\title{
Oscillation of half-linear differential equations with asymptotically almost periodic coefficients
}

Petr Hasil ${ }^{1 *}$ and Michal Veselý ${ }^{2}$

${ }^{*}$ Correspondence: hasil@mendelu.cz

${ }^{1}$ Department of Mathematics, Mendel University in Brno, Zemědělská 1, Brno, CZ-613 00, Czech Republic

Full list of author information is available at the end of the article

\begin{abstract}
We investigate second-order half-linear differential equations with asymptotically almost periodic coefficients. For these equations, we explicitly find an oscillation constant. If the coefficients are replaced by constants, our main result (concerning the conditional oscillation) reduces to the classical one. We also mention examples and concluding remarks.

MSC: 34C10; 34C15

Keywords: half-linear equations; oscillation theory; almost periodicity; asymptotic almost periodicity; conditional oscillation; oscillation constant
\end{abstract}

\section{Introduction}

This paper is devoted to the study of the half-linear differential equation

$$
\left[r(t) \Phi\left(x^{\prime}\right)\right]^{\prime}+c(t) \Phi(x)=0, \quad \Phi(x)=|x|^{p-1} \operatorname{sgn} x,
$$

where $r, c$ are continuous functions, $r$ is positive, and $p>1$. An equation of this form appeared for the first time in [1]. Nevertheless, as the basic pioneering papers in the field of half-linear differential equations, we mention $[2,3]$. During the last decades, these equations have been widely studied in the literature. A detailed description and a comprehensive literature overview concerning the topic can be found in [4] (see also [5, Chapter 3]).

The name half-linear equations was introduced in [6]. This term is motivated by the fact that the solution space of these equations is homogeneous (likewise in the linear case), but it is not additive. There are several differences between linear equations and halflinear equations. Especially, some tools widely used in the theory of linear equations are not available for half-linear equations (e.g., see [7] for the Wronskian identity and [8] for the Fredholm alternative). In fact, these differences are caused, more or less, by the lack of the additivity. On the other hand, many results from the theory of linear equations are extendable to their half-linear counterparts. Since the linear Sturmian theory extends verbatim to the half-linear case (for details, we refer to [4, Section 1.2]), we can classify Eq. (1.1) as oscillatory or non-oscillatory. We say that Eq. (1.1) is oscillatory if zeros of any solution tend to infinity. Otherwise, Eq. (1.1) is called non-oscillatory.

In this paper, we analyze a topic from the theory of half-linear equations and, at the same time, from the theory of (asymptotically) almost periodic functions. The importance of

(c) 2013 Hasil and Veselý: licensee Springer. This is an Open Access article distributed under the terms of the Creative Commons Attribution License (http://creativecommons.org/licenses/by/2.0), which permits unrestricted use, distribution, and reproduction in any medium, provided the original work is properly cited. 
the investigation of half-linear equations lies among others in the fact that various phenomena in physics and chemistry are studied through half-linear equations and through partial differential equations with $p$-Laplacian (half-linear equations can be regarded as equations with one-dimensional $p$-Laplacian). We refer to [9] and the last section of this paper (with references given therein). Similarly, many phenomena in nature have an oscillatory character and their models lead to the research of asymptotically almost periodic functions. There exist many remarkable books concerning (asymptotically) almost periodic differential equations and their applications. See, e.g., [10-12].

Actually, we are interested in the conditional oscillation of half-linear differential equations with asymptotically almost periodic coefficients. We say that the equation

$$
\left[r(t) \Phi\left(x^{\prime}\right)\right]^{\prime}+\gamma c(t) \Phi(x)=0
$$

with positive coefficients is conditionally oscillatory if there exists a constant $\Gamma>0$ such that Eq. (1.2) is oscillatory for all $\gamma>\Gamma$ and non-oscillatory for $\gamma<\Gamma$. The constant $\Gamma$ is called an oscillation constant (more precisely, oscillation constant of $c$ with respect to $r$ ). For results about the conditional oscillation of half-linear equations, we refer to $[13,14]$ and to [4, Section 5.4.2] (see also [15]). The conditional oscillation of linear equations is studied, e.g., in [16, 17].

Our goal is to find the explicit oscillation constant for Eq. (1.2), where $c(t)=t^{-p} s(t)$ and $r, s$ are asymptotically almost periodic functions. We point out that the main motivation of our research comes from $[14,17,18]$. In $[17,18]$, the oscillation constant is found for linear equations with periodic coefficients. Using the notion of the principal solution, the main result of [17] is generalized in [14], where periodic half-linear equations are considered. For the discrete counterpart, we refer to [19], where the problem is solved for linear difference equations with almost periodic coefficients. It means that the presented result is stronger than the known one in the discrete case because we consider asymptotically almost periodic half-linear equations.

We consider the asymptotically almost periodic functions $r, s$ in Eq. (1.2), because the known results do not cover two important cases - the almost periodic case, which is the basic generalization of the pure periodicity in applications, and perturbations vanishing at infinity. In this sense, our result is superior to the results of $[14,17,18]$.

Besides already given references, let us mention a short literature overview connected to the above announced result. The conditional oscillation of linear equations is treated in [16]. We also refer to [20-23] which generalize [16]. An oscillation criterion for secondorder half-linear equations is proved, e.g., in [24]. The oscillation properties of secondorder linear dynamic equations are studied in [25] and the oscillation and non-oscillation behavior of second-order half-linear dynamic equations is investigated in [26-28].

The oscillation of solutions of almost periodic linear equations is discussed in [29-31]. In [32], half-linear equations with (the Besicovitch) almost periodic coefficients are considered and an oscillation theorem is obtained. In [33], a necessary and sufficient condition for a second-order equation with the Besicovitch almost periodic coefficient to be oscillatory is given. Oscillation properties of these equations are mentioned in [5, Section 3.5] and [4, Section 5.3.3] as well. Other properties of half-linear equations with almost periodic coefficients are treated, e.g., in [34].

The paper is organized as follows. The notion of asymptotic almost periodicity is recalled in the next section. Necessary background from the theory of half-linear equations 
is presented in Section 3. In these two sections, we mention only basic definitions and results which we will use later. In Section 4, we prove the main result and we illustrate it by some examples. Our paper is finished by concluding remarks given in Section 5 .

Henceforth, for given $p>1$, the symbol $q$ will denote the number which is conjugated with $p$, i.e., $p+q=p q$. As usually, $\mathbb{R}^{+}$stands for all positive reals and $\mathbb{R}_{0}^{+}:=\mathbb{R}^{+} \cup\{0\}$.

\section{Asymptotic almost periodicity}

At first, we recall the definition of almost periodicity.

Definition 1 A continuous function $f: \mathbb{R} \rightarrow \mathbb{R}$ is called almost periodic if for every $\varepsilon>0$, there exists a number $p(\varepsilon)>0$ with the property that any interval of length $p(\varepsilon)$ of the real line contains at least one point $s$ for which

$$
|f(t+s)-f(t)|<\varepsilon, \quad t \in \mathbb{R}
$$

Note that we mentioned the so-called Bohr definition of almost periodic functions and that it is possible to introduce almost periodic functions in different ways. As the basic definition of almost periodic functions in the literature, one can often find the Bochner definition which is equivalent with the Bohr one and which is embodied in the next theorem.

Theorem 2.1 Let $f: \mathbb{R} \rightarrow \mathbb{R}$ be a continuous function. Then $f$ is almost periodic if and only iffrom any sequence of the form $\left\{f\left(t+s_{n}\right)\right\}_{n \in \mathbb{N}}$, where $s_{n}$ are real numbers, one can extract a subsequence which converges uniformly with respect to $t \in \mathbb{R}$.

Proof See, e.g., [12, Theorem 1.14].

As a direct generalization of almost periodicity, we consider the notion of asymptotic almost periodicity.

Definition 2 We say that a continuous function $f: \mathbb{R}_{0}^{+} \rightarrow \mathbb{R}$ is asymptotically almost periodic if it can be represented in the form $f(t)=f_{1}(t)+f_{2}(t), t \in \mathbb{R}_{0}^{+}$, where $f_{1}$ is almost periodic and $f_{2}$ has the property that $\lim _{t \rightarrow \infty} f_{2}(t)=0$.

Again, equivalent definitions of asymptotic almost periodicity (based on the Bohr and Bochner concept) are used in the literature. We formulate them in the following two theorems. For the proofs of these theorems, we refer to [12, Theorem 9.3, parts 2), 4)] (see also [10, Theorem 1.5.2]).

Theorem 2.2 A continuous function $f: \mathbb{R}_{0}^{+} \rightarrow \mathbb{R}$ is asymptotically almost periodic if and only iffor every $\varepsilon>0$, there exist numbers $p(\varepsilon)>0$ and $P(\varepsilon)>0$ with the property that any interval $I \subset \mathbb{R}$ of length $p(\varepsilon)$ contains at least one point $s$ for which

$$
|f(t+s)-f(t)|<\varepsilon, \quad t, t+s \geq P(\varepsilon)
$$

Theorem 2.3 A continuous function $f: \mathbb{R}_{0}^{+} \rightarrow \mathbb{R}$ is asymptotically almost periodic if and only if from any sequence of the form $\left\{f\left(t+s_{n}\right)\right\}_{n \in \mathbb{N}}$, where $s_{n}$ are positive real numbers 
satisfying $\lim _{n \rightarrow \infty} s_{n}=\infty$, one can extract a subsequence which converges uniformly with respect to $t \in \mathbb{R}_{0}^{+}$.

Now we mention two well-known common properties of almost periodic and asymptotically almost periodic functions, which we will need later. To prove them, it suffices to consider Theorems 2.1 and 2.3 (or consider, e.g., the proof of [12, Theorem 1.9]).

Corollary 2.1 Iff is an (asymptotically) almost periodic function and $F: \mathbb{R} \rightarrow \mathbb{R}$ is uniformly continuous on the range of $f$, then the function $F \circ f$ is (asymptotically) almost periodic as well.

Corollary 2.2 The sum and the product of two (asymptotically) almost periodic functions are (asymptotically) almost periodic.

Since asymptotically almost periodic functions are evidently bounded, from Corollary 2.1, we also obtain the following.

Corollary 2.3 Iff is an asymptotically almost periodic function satisfying

$$
\inf \left\{f(t) ; t \in \mathbb{R}_{0}^{+}\right\}>0,
$$

then the function $g: \mathbb{R}_{0}^{+} \rightarrow \mathbb{R}^{+}$, defined by the formula $g(t)=f^{\alpha}(t)$, is asymptotically almost periodic for arbitrarily given $\alpha \in \mathbb{R}$.

It remains to recall the notion of the mean value for asymptotically almost periodic functions.

Theorem 2.4 Iff is an asymptotically almost periodic function, then the limit

$$
M(f):=\lim _{t \rightarrow \infty} \frac{1}{t} \int_{a}^{a+t} f(s) \mathrm{d} s
$$

is finite and exists uniformly with respect to $a \in \mathbb{R}_{0}^{+}$.

Proof The theorem follows, e.g., from [11, Theorem 3.4] (see also [11, Remark 3.15]).

Definition 3 Let $f$ be asymptotically almost periodic. The number $M(f)$ introduced in (2.1) is called the mean value of $f$.

Remark 1 For any positive asymptotically almost periodic function $f$ with the property that lim $\sup _{t \rightarrow \infty} f(t)>0$, we have $M(f)>0$. To verify this fact, it suffices to use Theorem 2.2.

\section{Half-linear differential equations}

To prove the main result, we use the concept of the Riccati equation for half-linear equations. More precisely, applying the transformation

$$
w(t)=r(t) \Phi\left(\frac{x^{\prime}(t)}{x(t)}\right), \quad \Phi(x)=|x|^{p-1} \operatorname{sgn} x,
$$


to Eq. (1.1), we obtain the half-linear Riccati equation

$$
w^{\prime}(t)+c(t)+(p-1) r^{1-q}(t)|w(t)|^{q}=0
$$

whenever $x(t) \neq 0$. We remark that the connection between Eq. (1.1) and Eq. (3.1) is described in the below given Theorem 3.1.

We also need the following two theorems. The first one is a well-known consequence of the half-linear Roundabout theorem which is proved, e.g., in [4, Theorem 1.2.2] (or see directly [4, Theorem 2.2.1]). For the second one, we can refer to [4, Theorem 2.2.3].

Theorem 3.1 Equation (1.1) is non-oscillatory if and only if there exists a function $w$ which solves Eq. (3.1) on some interval $[T, \infty)$.

Theorem 3.2 Suppose that

$$
\int^{\infty} r^{1-q}(t) \mathrm{d} t=\infty
$$

and that Eq. (1.1) is non-oscillatory. Then the lower limit

$$
\liminf _{t \rightarrow \infty} \frac{\int^{t} r^{1-q}(\varphi)\left(\int^{\varphi} c(\tau) \mathrm{d} \tau\right) \mathrm{d} \varphi}{\int^{t} r^{1-q}(\varphi) \mathrm{d} \varphi}>-\infty
$$

if and only if

$$
\int^{\infty} r^{1-q}(t)|w(t)|^{q} \mathrm{~d} t<\infty
$$

for every solution $w$ of $E q$. (3.1).

We focus our attention to Eq. (1.1) in the form

$$
\left[r(t) \Phi\left(x^{\prime}\right)\right]^{\prime}+\gamma \frac{s(t)}{t^{p}} \Phi(x)=0,
$$

where $\gamma \in \mathbb{R}^{+}$and $r, s$ are positive asymptotically almost periodic functions. The Riccati equation associated to Eq. (3.5) has the form

$$
w^{\prime}(t)+\gamma \frac{s(t)}{t^{p}}+(p-1) r^{1-q}(t)|w(t)|^{q}=0 .
$$

For $\zeta(t)=-w(t) t^{p-1}$, we express

$$
\begin{aligned}
\zeta^{\prime}(t) & =\left[-w(t) t^{p-1}\right]^{\prime}=-\left[w^{\prime}(t) t^{p-1}+(p-1) w(t) t^{p-2}\right] \\
& =\frac{1}{t}\left[(p-1) \zeta(t)+\gamma s(t)+(p-1) \frac{|\zeta(t)|^{q}}{r^{q-1}(t)}\right],
\end{aligned}
$$

i.e., we obtain the so-called adapted Riccati equation

$$
\zeta^{\prime}(t)=\frac{1}{t}\left[(p-1) \zeta(t)+\gamma s(t)+(p-1) \frac{|\zeta(t)|^{q}}{r^{q-1}(t)}\right] .
$$


In fact, we will use only partial cases of Theorems 3.1 and 3.2. For reader's convenience, we mention the corresponding corollaries for Eq. (3.5) and Eq. (3.7).

Corollary 3.1 Let Eq. (3.5) be non-oscillatory. There exists a negative solution $\zeta$ of the adapted Riccati equation (3.7) on some interval $\left[t_{0}, \infty\right)$.

Proof We apply Theorem 3.2, where (3.2) is satisfied (consider the boundedness of $r$ ). Inequality (3.3) is valid, because $r, s$ are positive functions and $\gamma \in \mathbb{R}^{+}$. Thus, (3.4) holds for all solutions $w$ of Eq. (3.6). Let us consider an arbitrary solution $w$ of Eq. (3.6) defined on $\left[t_{0}, \infty\right.$ ) satisfying $w\left(t_{0}\right)>0$ (see Theorem 3.1). We know that $w$ is decreasing which follows immediately from (3.6). By combination of (3.2) and (3.4), we obtain $w(t) \rightarrow 0$ as $t \rightarrow \infty$. Especially, $w(t)>0, t \geq t_{0}$. This observation implies the existence of a negative solution $\zeta$ of Eq. (3.7) on $\left[t_{0}, \infty\right)$.

Corollary 3.2 If there exists a negative function $\zeta$ which solves the adapted Riccati equation (3.7) on some interval $[T, \infty)$, then Eq. (3.5) is non-oscillatory.

Proof The statement of the corollary follows directly from Theorem 3.1.

\section{Oscillation constant}

Now we can formulate and prove the announced result.

Theorem 4.1 Let $\gamma \in \mathbb{R}^{+}$be given and let $r$ and $s$ be arbitrary positive asymptotically almost periodic functions satisfying

$$
\inf \left\{r(t) ; t \in \mathbb{R}_{0}^{+}\right\}>0, \quad \quad \limsup _{t \rightarrow \infty} s(t)>0 .
$$

Let

$$
\Gamma:=q^{-p}\left[M\left(r^{1-q}\right)\right]^{1-p}[M(s)]^{-1}
$$

Consider the equation

$$
\left[r(t) \Phi\left(x^{\prime}\right)\right]^{\prime}+\gamma \frac{s(t)}{t^{p}} \Phi(x)=0, \quad \Phi(x)=|x|^{p-1} \operatorname{sgn} x .
$$

If $\gamma>\Gamma$, then Eq. (4.3) is oscillatory. If $\gamma<\Gamma$, then Eq. (4.3) is non-oscillatory.

Proof In the both cases of the theorem, we consider $\gamma \neq \Gamma$. Let $\alpha, T \in \mathbb{R}^{+}$be such that

$$
\begin{aligned}
& \frac{1}{\alpha} \int_{t}^{t+\alpha} s(\tau) \mathrm{d} \tau>\frac{M(s)}{2}>0, \quad t \geq T, \\
& \left|\Gamma-q^{-p}\left(\frac{1}{\alpha} \int_{t}^{t+\alpha} \frac{1}{r^{q-1}(\tau)} \mathrm{d} \tau\right)^{-\frac{p}{q}}\left(\frac{1}{\alpha} \int_{t}^{t+\alpha} s(\tau) \mathrm{d} \tau\right)^{-1}\right|<\frac{|\Gamma-\gamma|}{2}, \quad t \geq T,
\end{aligned}
$$

i.e.,

$$
\left|\gamma-q^{-p}\left(\frac{1}{\alpha} \int_{t}^{t+\alpha} \frac{1}{r^{q-1}(\tau)} \mathrm{d} \tau\right)^{-\frac{p}{q}}\left(\frac{1}{\alpha} \int_{t}^{t+\alpha} s(\tau) \mathrm{d} \tau\right)^{-1}\right|>\frac{|\gamma-\Gamma|}{2}, \quad t \geq T .
$$


The existence of such numbers follows from Corollary 2.3, Theorem 2.4, and Remark 1. We denote

$$
\begin{aligned}
& r^{+}:=\sup \left\{r(t) ; t \in \mathbb{R}_{0}^{+}\right\}, \quad s^{+}:=\sup \left\{s(t) ; t \in \mathbb{R}_{0}^{+}\right\}, \\
& r^{-}:=\inf \left\{r(t) ; t \in \mathbb{R}_{0}^{+}\right\}>0 .
\end{aligned}
$$

Let $\gamma>\Gamma$. By contradiction, we suppose that Eq. (4.3) is non-oscillatory. Corollary 3.1 says that the adapted Riccati equation has a negative solution $\zeta$, which exists on some interval $\left[t_{0}, \infty\right)$. Without loss of generality, we can assume that $t_{0}=T$. We recall (see (3.7))

$$
\zeta^{\prime}(t)=\frac{1}{t}\left[(p-1) \zeta(t)+\gamma s(t)+(p-1) \frac{|\zeta(t)|^{q}}{r^{q-1}(t)}\right], \quad t>t_{0}
$$

Especially, we know that

$$
\zeta^{\prime}(t)>\frac{p-1}{t}\left[\zeta(t)+\frac{|\zeta(t)|^{q}}{\left(r^{+}\right)^{q-1}}\right], \quad t>t_{0}
$$

Since $q>1$, from (4.9) it follows the existence of $K>0$ for which

$$
\zeta(t) \in(-K, 0), \quad t \geq t_{0} .
$$

Indeed, $\zeta^{\prime}(t)>0$ if we assume that $\zeta(t) \leq-r^{+}$.

In addition, considering (4.8) and (4.10), we have

$$
\int_{t}^{t+a}\left|\zeta^{\prime}(\tau)\right| \mathrm{d} \tau \leq \frac{p-1}{t} \int_{t}^{t+a}|\zeta(\tau)|+\frac{|\zeta(\tau)|^{q}}{\left(r^{-}\right)^{q-1}} \mathrm{~d} \tau+\frac{a \gamma s^{+}}{t} \leq \frac{C a}{t}, \quad t \geq t_{0}, a>0,
$$

where

$$
C:=(p-1)\left[K+\frac{K^{q}}{\left(r^{-}\right)^{q-1}}\right]+\gamma s^{+} .
$$

Therefore,

$$
\left|\zeta\left(t_{2}\right)-\zeta\left(t_{1}\right)\right| \leq \int_{t_{1}}^{t_{2}}\left|\zeta^{\prime}(\tau)\right| \mathrm{d} \tau \leq \frac{C\left(t_{2}-t_{1}\right)}{t_{1}}, \quad t_{2} \geq t_{1} \geq t_{0}
$$

i.e.,

$$
\begin{aligned}
|\zeta(t+\sigma)-\zeta(t+\tau)| & \leq \frac{C|t+\sigma-t-\tau|}{t} \\
& =\frac{C|\tau-\sigma|}{t} \leq \frac{C \alpha}{t}, \quad \tau, \sigma \in[0, \alpha], t \geq t_{0} .
\end{aligned}
$$

We denote

$$
\xi(t):=\frac{1}{\alpha} \int_{t}^{t+\alpha} \zeta(\tau) \mathrm{d} \tau, \quad t \geq t_{0}
$$


Since (4.13) holds for all $\sigma \in[0, \alpha]$, we obtain

$$
|\xi(t)-\zeta(t+\tau)| \leq \frac{C \alpha}{t}, \quad t \geq t_{0}, \tau \in[0, \alpha]
$$

We also put

$$
\begin{aligned}
& X(t):=(p-1)\left(\frac{p}{\alpha} \int_{t}^{t+\alpha} \frac{1}{r^{q-1}(\tau)} \mathrm{d} \tau\right)^{-\frac{1}{q}}, \quad t \geq t_{0}, \\
& Y(t):=|\xi(t)|\left(\frac{p}{\alpha} \int_{t}^{t+\alpha} \frac{1}{r^{q-1}(\tau)} \mathrm{d} \tau\right)^{\frac{1}{q}}, \quad t \geq t_{0} .
\end{aligned}
$$

Henceforth (in this part of the proof), we will consider $t \geq t_{0}$. We have

$$
\begin{aligned}
\xi^{\prime}(t)= & \frac{1}{\alpha} \int_{t}^{t+\alpha} \zeta^{\prime}(\tau) \mathrm{d} \tau=\frac{1}{\alpha} \int_{t}^{t+\alpha} \frac{1}{\tau}\left[(p-1) \zeta(\tau)+\gamma s(\tau)+(p-1) \frac{|\zeta(\tau)|^{q}}{r^{q-1}(\tau)}\right] \mathrm{d} \tau \\
\geq & \frac{1}{t+\alpha}\left[(p-1) \xi(t)+\frac{\gamma}{\alpha} \int_{t}^{t+\alpha} s(\tau) \mathrm{d} \tau+\frac{p-1}{\alpha} \int_{t}^{t+\alpha} \frac{|\zeta(\tau)|^{q}}{r^{q-1}(\tau)} \mathrm{d} \tau\right] \\
= & \frac{1}{t+\alpha}\left[(p-1) \xi(t)+\frac{X^{p}(t)}{p}+\frac{Y^{q}(t)}{q}+\frac{\gamma}{\alpha} \int_{t}^{t+\alpha} s(\tau) \mathrm{d} \tau-\frac{X^{p}(t)}{p}\right. \\
& \left.+\frac{p-1}{\alpha} \int_{t}^{t+\alpha} \frac{|\zeta(\tau)|^{q}}{r^{q-1}(\tau)} \mathrm{d} \tau-\frac{Y^{q}(t)}{q}\right]
\end{aligned}
$$

if $\xi^{\prime}(t)>0$ for an arbitrarily given $t \geq t_{0}$, or

$$
\begin{aligned}
\xi^{\prime}(t) \geq & \frac{1}{t}\left[(p-1) \xi(t)+\frac{X^{p}(t)}{p}+\frac{Y^{q}(t)}{q}+\frac{\gamma}{\alpha} \int_{t}^{t+\alpha} s(\tau) \mathrm{d} \tau-\frac{X^{p}(t)}{p}\right. \\
& \left.+\frac{p-1}{\alpha} \int_{t}^{t+\alpha} \frac{|\zeta(\tau)|^{q}}{r^{q-1}(\tau)} \mathrm{d} \tau-\frac{Y^{q}(t)}{q}\right]
\end{aligned}
$$

if $\xi^{\prime}(t)<0$.

Since

$$
(p-1) \xi(t)=-(p-1)|\xi(t)|=-X(t) Y(t),
$$

we obtain

$$
(p-1) \xi(t)+\frac{X^{p}(t)}{p}+\frac{Y^{q}(t)}{q}=\frac{X^{p}(t)}{p}+\frac{Y^{q}(t)}{q}-X(t) Y(t) \geq 0,
$$

which follows from Young's inequality.

Next, applying (4.4), (4.5), and (4.6), we compute

$$
\begin{aligned}
& \frac{\gamma}{\alpha} \int_{t}^{t+\alpha} s(\tau) \mathrm{d} \tau-\frac{X^{p}(t)}{p} \\
& \quad=\frac{\gamma}{\alpha} \int_{t}^{t+\alpha} s(\tau) \mathrm{d} \tau-\frac{(p-1)^{p}}{p}\left(\frac{p}{\alpha} \int_{t}^{t+\alpha} \frac{1}{r^{q-1}(\tau)} \mathrm{d} \tau\right)^{-\frac{p}{q}}
\end{aligned}
$$




$$
\begin{aligned}
& =\frac{\gamma}{\alpha} \int_{t}^{t+\alpha} s(\tau) \mathrm{d} \tau-q^{-p}\left(\frac{1}{\alpha} \int_{t}^{t+\alpha} \frac{1}{r^{q-1}(\tau)} \mathrm{d} \tau\right)^{-\frac{p}{q}} \frac{\frac{1}{\alpha} \int_{t}^{t+\alpha} s(\tau) \mathrm{d} \tau}{\frac{1}{\alpha} \int_{t}^{t+\alpha} s(\tau) \mathrm{d} \tau} \\
& =\frac{1}{\alpha} \int_{t}^{t+\alpha} s(\tau) \mathrm{d} \tau\left[\gamma-q^{-p}\left(\frac{1}{\alpha} \int_{t}^{t+\alpha} \frac{1}{r^{q-1}(\tau)} \mathrm{d} \tau\right)^{-\frac{p}{q}}\left(\frac{1}{\alpha} \int_{t}^{t+\alpha} s(\tau) \mathrm{d} \tau\right)^{-1}\right] \\
& >\frac{M(s)}{2} \frac{\gamma-\Gamma}{2}>0,
\end{aligned}
$$

i.e.,

$$
\frac{\gamma}{\alpha} \int_{t}^{t+\alpha} s(\tau) \mathrm{d} \tau-\frac{X^{p}(t)}{p}>M(s) \frac{\gamma-\Gamma}{4} .
$$

The function $y=|x|^{q}$ is continuously differentiable on $[-K, 0]$, and hence there exists $L>0$ satisfying

$$
\left.|| \xi\right|^{q}-|\zeta|^{q}|\leq L| \xi-\zeta \mid, \quad \xi, \zeta \in[-K, 0]
$$

Using the fact that this function has the Lipschitz property and using (4.14), we obtain

$$
\begin{aligned}
& \frac{p-1}{\alpha} \int_{t}^{t+\alpha} \frac{|\zeta(\tau)|^{q}}{r^{q-1}(\tau)} \mathrm{d} \tau-\frac{Y^{q}(t)}{q} \\
& =\frac{p-1}{\alpha} \int_{t}^{t+\alpha} \frac{|\zeta(\tau)|^{q}}{r^{q-1}(\tau)} \mathrm{d} \tau-\frac{|\xi(t)|^{q}}{q} \frac{q(p-1)}{\alpha} \int_{t}^{t+\alpha} \frac{1}{r^{q-1}(\tau)} \mathrm{d} \tau \\
& =-\frac{p-1}{\alpha} \int_{t}^{t+\alpha} \frac{|\xi(t)|^{q}-|\zeta(\tau)|^{q}}{r^{q-1}(\tau)} \mathrm{d} \tau \geq-\frac{p-1}{\alpha} \frac{1}{\left(r^{-}\right)^{q-1}} \int_{t}^{t+\alpha} L|\xi(t)-\zeta(\tau)| \mathrm{d} \tau \\
& \geq-\frac{p-1}{\alpha} \frac{1}{\left(r^{-}\right)^{q-1}} \alpha L \frac{C \alpha}{t}=-\frac{C L \alpha(p-1)\left(r^{-}\right)^{1-q}}{t} .
\end{aligned}
$$

For sufficiently large $t \geq t_{0}$, it is valid

$$
\frac{p-1}{\alpha} \int_{t}^{t+\alpha} \frac{|\zeta(\tau)|^{q}}{r^{q-1}(\tau)} \mathrm{d} \tau-\frac{Y^{q}(t)}{q}>-M(s) \frac{\gamma-\Gamma}{8}
$$

Altogether (consider (4.17), (4.18), (4.19), (4.20)), there exists $\hat{t} \geq t_{0}$ for which we have

$$
\xi^{\prime}(t)>\frac{1}{t+\alpha}\left[0+M(s) \frac{\gamma-\Gamma}{4}-M(s) \frac{\gamma-\Gamma}{8}\right]=\frac{1}{t+\alpha} M(s) \frac{\gamma-\Gamma}{8}, \quad t \geq \hat{t}
$$

which implies that

$$
\xi(t) \rightarrow \infty \quad \text { as } t \rightarrow \infty, \quad \text { i.e., } \quad \limsup _{t \rightarrow \infty} \zeta(t)=\infty
$$

This contradiction proves that Eq. (4.3) is oscillatory for $\gamma>\Gamma$.

In the second part of the proof, we have to show that Eq. (4.3) is non-oscillatory for $\gamma<\Gamma$. We will consider arbitrarily given $\gamma<\Gamma$ and the solution $\zeta$ of the adapted Riccati equation determined by the Cauchy problem (4.8), $\zeta(T)=2 \xi_{0}$, where

$$
\xi_{0}:=-\left[\frac{p}{\alpha(p-1)} \int_{T}^{T+\alpha} \frac{1}{r^{q-1}(\tau)} \mathrm{d} \tau\right]^{1-p}
$$


As in the first part of the proof (see (4.9)), we can estimate

$$
\zeta^{\prime}(t)>\frac{p-1}{t}\left[\zeta(t)+\frac{|\zeta(t)|^{q}}{\left(r^{+}\right)^{q-1}}\right]
$$

for all $t$ from the domain of $\zeta$. Thus, there exists $K>0$ such that $\zeta(t)>-K$ for all considered $t$. Analogously (see (4.12)), we can also prove

$$
\begin{aligned}
\left|\zeta\left(t_{2}\right)-\zeta\left(t_{1}\right)\right| & \leq \int_{t_{1}}^{t_{2}}\left|\zeta^{\prime}(\tau)\right| \mathrm{d} \tau \\
& \leq \frac{(p-1)\left[K+\frac{K^{q}}{\left(r^{-}\right)^{q-1}}\right]+\gamma s^{+}}{T}\left(t_{2}-t_{1}\right), \quad t_{2} \geq t_{1} \geq T
\end{aligned}
$$

if $\zeta\left(t_{1}\right), \zeta\left(t_{2}\right)<0$. Hence, we can assume that $T$ is so large that it is true

$$
\left|\zeta\left(t_{2}\right)-\zeta\left(t_{1}\right)\right|<-\frac{\xi_{0}}{2}, \quad t_{1}+\alpha \geq t_{2} \geq t_{1} \geq T
$$

if $\zeta\left(t_{1}\right), \zeta\left(t_{2}\right)<0$. Especially, provided that $\zeta(t) \leq \xi_{0} / 2$ for some $t \geq T$, we have $\zeta(\tau)<0$ for all $\tau \in[t, t+\alpha]$. Thus, we can define

$$
\xi(t):=\frac{1}{\alpha} \int_{t}^{t+\alpha} \zeta(\tau) \mathrm{d} \tau
$$

for all considered $t \geq T$ such that $\zeta(t) \leq \xi_{0} / 2$. From (4.21) it follows

$$
|\xi(t)-\zeta(t+\tau)| \leq \frac{C \alpha}{T}
$$

for all considered $t \geq T$ and $\tau \in[0, \alpha]$, where $C$ is introduced in (4.11).

We prove that $\zeta(t) \leq-\delta$ for some $\delta>0$ and for all considered $t \geq T$. On the contrary, we will assume that such a number $\delta$ does not exist. This assumption implies the existence of $t_{1}>T$ such that $\xi^{\prime}\left(t_{1}\right)>0$ and $\xi\left(t_{1}\right)=\xi_{0}$. We estimate the value $\xi^{\prime}\left(t_{1}\right)$ by the following expression:

$$
\begin{aligned}
\xi^{\prime}\left(t_{1}\right)= & \frac{1}{\alpha} \int_{t_{1}}^{t_{1}+\alpha} \frac{1}{\tau}\left[(p-1) \zeta(\tau)+\gamma s(\tau)+(p-1) \frac{|\zeta(\tau)|^{q}}{r^{q-1}(\tau)}\right] \mathrm{d} \tau \\
\leq & \frac{1}{t_{1}}\left[(p-1) \xi\left(t_{1}\right)+\frac{\gamma}{\alpha} \int_{t_{1}}^{t_{1}+\alpha} s(\tau) \mathrm{d} \tau+\frac{p-1}{\alpha} \int_{t_{1}}^{t_{1}+\alpha} \frac{|\zeta(\tau)|^{q}}{r^{q-1}(\tau)} \mathrm{d} \tau\right] \\
= & \frac{1}{t_{1}}\left[(p-1) \xi\left(t_{1}\right)+\frac{X^{p}\left(t_{1}\right)}{p}+\frac{Y^{q}\left(t_{1}\right)}{q}+\frac{\gamma}{\alpha} \int_{t_{1}}^{t_{1}+\alpha} s(\tau) \mathrm{d} \tau-\frac{X^{p}\left(t_{1}\right)}{p}\right. \\
& \left.+\frac{p-1}{\alpha} \int_{t_{1}}^{t_{1}+\alpha} \frac{|\zeta(\tau)|^{q}}{r^{q-1}(\tau)} \mathrm{d} \tau-\frac{Y^{q}\left(t_{1}\right)}{q}\right]
\end{aligned}
$$

where $X\left(t_{1}\right)$ and $Y\left(t_{1}\right)$ are defined in (4.15) and (4.16), respectively.

Assume that

$$
\frac{1}{\alpha} \int_{T}^{T+\alpha} \frac{1}{r^{q-1}(\tau)} \mathrm{d} \tau=\frac{1}{\alpha} \int_{t_{1}}^{t_{1}+\alpha} \frac{1}{r^{q-1}(\tau)} \mathrm{d} \tau .
$$


In this case, considering $X^{p}\left(t_{1}\right)=Y^{q}\left(t_{1}\right)$, we have

$$
\begin{aligned}
\frac{X^{p}\left(t_{1}\right)}{p}+\frac{Y^{q}\left(t_{1}\right)}{q} & =X^{p}\left(t_{1}\right)\left(\frac{1}{p}+\frac{1}{q}\right)=X^{1+\frac{p}{q}}\left(t_{1}\right) \\
& =X\left(t_{1}\right)\left(Y^{q}\left(t_{1}\right)\right)^{\frac{1}{q}}=X\left(t_{1}\right) Y\left(t_{1}\right)=-(p-1) \xi\left(t_{1}\right)
\end{aligned}
$$

and, consequently, we obtain

$$
(p-1) \xi\left(t_{1}\right)+\frac{X^{p}\left(t_{1}\right)}{p}+\frac{Y^{q}\left(t_{1}\right)}{q}=0 .
$$

We consider $T, \alpha$ as sufficiently large numbers for which (4.4) and (4.5) are satisfied. Analogously, we can require that $T$ and $\alpha$ are so large that

$$
\left|\frac{1}{\alpha} \int_{T}^{T+\alpha} \frac{1}{r^{q-1}(\tau)} \mathrm{d} \tau-\frac{1}{\alpha} \int_{t_{1}}^{t_{1}+\alpha} \frac{1}{r^{q-1}(\tau)} \mathrm{d} \tau\right|<\varepsilon
$$

for arbitrarily given $\varepsilon>0$. Note that $t_{1}>T$. Thus, for sufficiently large $T$, we can assume that

$$
(p-1) \xi\left(t_{1}\right)+\frac{X^{p}\left(t_{1}\right)}{p}+\frac{Y^{q}\left(t_{1}\right)}{q}<\frac{M(s)(\Gamma-\gamma)}{16} .
$$

We compute

$$
\begin{aligned}
& \frac{\gamma}{\alpha} \int_{t_{1}}^{t_{1}+\alpha} s(\tau) \mathrm{d} \tau-\frac{X^{p}\left(t_{1}\right)}{p} \\
& \quad=\frac{\gamma}{\alpha} \int_{t_{1}}^{t_{1}+\alpha} s(\tau) \mathrm{d} \tau-\frac{(p-1)^{p}}{p}\left(\frac{p}{\alpha} \int_{t_{1}}^{t_{1}+\alpha} \frac{1}{r^{q-1}(\tau)} \mathrm{d} \tau\right)^{-\frac{p}{q}} \\
& \quad=\frac{1}{\alpha} \int_{t_{1}}^{t_{1}+\alpha} s(\tau) \mathrm{d} \tau\left[\gamma-q^{-p}\left(\frac{1}{\alpha} \int_{t_{1}}^{t_{1}+\alpha} \frac{1}{r^{q-1}(\tau)} \mathrm{d} \tau\right)^{-\frac{p}{q}}\left(\frac{1}{\alpha} \int_{t_{1}}^{t_{1}+\alpha} s(\tau) \mathrm{d} \tau\right)^{-1}\right] .
\end{aligned}
$$

Therefore, using (4.4), (4.5), and (4.6), we have

$$
\frac{\gamma}{\alpha} \int_{t_{1}}^{t_{1}+\alpha} s(\tau) \mathrm{d} \tau-\frac{X^{p}\left(t_{1}\right)}{p}<\frac{M(s)}{2} \frac{\gamma-\Gamma}{2}<0 .
$$

Similarly as in the first part of the proof (see (4.22)), we can show

$$
\frac{p-1}{\alpha} \int_{t_{1}}^{t_{1}+\alpha} \frac{|\zeta(\tau)|^{q}}{r^{q-1}(\tau)} \mathrm{d} \tau-\frac{Y^{q}\left(t_{1}\right)}{q} \leq \frac{p-1}{T} \frac{C L \alpha}{\left(r^{-}\right)^{q-1}} .
$$

Assume that

$$
T>\frac{16(p-1)}{M(s)(\Gamma-\gamma)} \frac{C L \alpha}{\left(r^{-}\right)^{q-1}},
$$

which implies

$$
\frac{p-1}{\alpha} \int_{t_{1}}^{t_{1}+\alpha} \frac{|\zeta(\tau)|^{q}}{r^{q-1}(\tau)} \mathrm{d} \tau-\frac{Y^{q}\left(t_{1}\right)}{q}<\frac{M(s)(\Gamma-\gamma)}{16} .
$$


Finally, considering (4.23), (4.24), (4.25), and (4.26), we obtain the following contradiction:

$$
\xi^{\prime}\left(t_{1}\right)<\frac{M(s)(\gamma-\Gamma)}{8 t_{1}}<0
$$

which means that there exists $\delta>0$ satisfying

$$
\zeta(t) \leq-\delta, \quad t \geq T
$$

Especially, this negative solution exists for all $t \geq T$. From Corollary 3.2 it follows that Eq. (4.3) is non-oscillatory for $\gamma<\Gamma$.

Remark 2 Our result is 'backward compatible' with previous results about the conditional oscillation of half-linear equations. More precisely, if the functions $r$ and $s$ in Eq. (4.3) are $\alpha$-periodic ones (with the same period $\alpha$ ), our constant $\Gamma$ reduces to the one from [14]. If they are replaced by constants $r, s \in \mathbb{R}^{+}$, then $\Gamma=q^{-p} r / s$, which gives the classical result for half-linear Euler-type equations (see, e.g., [35] or [4, Section 1.4.2]).

Example 1 Let $a>0, \alpha, \beta \neq 0$, and $c>1 / 2$. We can use Theorem 4.1 for the equation

$$
\left[\frac{t^{2}}{1+t^{2}(\cos [a t] \sin [a t]+c)} \Phi\left(x^{\prime}\right)\right]^{\prime}+\gamma \frac{\arctan (\sin [\alpha t])+|\sin [\alpha t]|+|\cos [\beta t]|}{\sqrt{t^{3}}} \Phi(x)=0
$$

whose coefficients are asymptotically almost periodic (it suffices to consider directly Definition 2 and Corollaries 2.2, 2.3) and where $p=3 / 2$ (i.e., $q=3$ ). It holds

$$
\begin{aligned}
& M\left(r^{-2}\right)=M\left(\left(\cos [a t] \sin [a t]+c+\frac{1}{t^{2}}\right)^{2}\right)=\frac{8 c^{2}+1}{8}, \\
& M(s)=M(\arctan (\sin [\alpha t])+|\sin [\alpha t]|+|\cos [\beta t]|)=\frac{4}{\pi}
\end{aligned}
$$

Thus, the equation is oscillatory for $6 \gamma \sqrt{3\left(8 c^{2}+1\right)}>\sqrt{2} \pi$ and, at the same time, nonoscillatory for $6 \gamma \sqrt{3\left(8 c^{2}+1\right)}<\sqrt{2} \pi$.

The statement of Theorem 4.1 is a new result also for $p=2$ (even for linear equations with almost periodic coefficients).

Corollary 4.1 Consider the equation

$$
\left[r(t) x^{\prime}\right]^{\prime}+\gamma \frac{s(t)}{t^{2}} x=0
$$

where $\gamma \in \mathbb{R}^{+}$and $r$, s are positive almost periodic functions satisfying (4.1). Then the oscillation constant is

$$
\Gamma=\left[4 M\left(r^{-1}\right) M(s)\right]^{-1},
$$

i.e., Eq. (4.27) is oscillatory for $\gamma>\Gamma$ and non-oscillatory for $\gamma<\Gamma$. 
If the functions $r$ and $s$ in Eq. (4.27) are periodic with the same period, then the oscillation constant given in (4.28) reduces to the known one (see [17]). For constant functions $r$, $s$, we obtain $\Gamma=r /(4 s)$, which corresponds to the famous result of Kneser [36]. Note that the main result of [17] cannot be used for general periodic functions $r, s$ (which do not have any common period). This situation is illustrated by the following example.

Example 2 Let continuous functions $f, g:[-1,1] \rightarrow \mathbb{R}^{+}$be given. Let us consider the equation

$$
\left[f(\sin t) x^{\prime}\right]^{\prime}+\gamma \frac{g[\sin (\sqrt{2} t)]}{t^{2}} x=0
$$

The almost periodicity of the functions $r(t)=f(\sin t), s(t)=g[\sin (\sqrt{2} t)]$ is obvious. It is also seen that (4.1) is satisfied and that

$$
M\left(r^{-1}\right)=\frac{1}{2 \pi} \int_{-\pi}^{\pi} \frac{\mathrm{d} t}{f(\sin t)}, \quad M(s)=\frac{\sqrt{2}}{2 \pi} \int_{-\pi / \sqrt{2}}^{\pi / \sqrt{2}} g[\sin (\sqrt{2} t)] \mathrm{d} t .
$$

Hence, we obtain the oscillation constant

$$
\Gamma=\left[\frac{\sqrt{2}}{\pi^{2}} \int_{-\pi}^{\pi} \frac{\mathrm{d} t}{f(\sin t)} \int_{-\pi / \sqrt{2}}^{\pi / \sqrt{2}} g[\sin (\sqrt{2} t)] \mathrm{d} t\right]^{-1} .
$$

\section{Concluding remarks}

There exist positive almost periodic functions $f, g: \mathbb{R} \rightarrow \mathbb{R}$ for which

$$
\frac{1}{\alpha} \int_{0}^{\alpha} f(t) \mathrm{d} t<M(f), \quad \frac{1}{\alpha} \int_{0}^{\alpha} g(t) \mathrm{d} t>M(g), \quad \alpha>0 .
$$

Such functions can be constructed using, e.g., [37, Theorem 3.1]. Based on this fact, we conjecture that there exist almost periodic functions $r_{1}, s_{1}, r_{2}, s_{2}$ satisfying (4.1) such that Eq. (4.3) determined by the coefficients $r_{1}, s_{1}$ and $\gamma=\Gamma$ (see (4.2)) is oscillatory and Eq. (4.3) with the coefficients $r_{2}, s_{2}$ and $\gamma=\Gamma$ is non-oscillatory. Thus, in general, we conjecture that it is not possible to decide whether Eq. (4.3) is oscillatory or non-oscillatory for $\gamma=\Gamma$.

The statement of Theorem 4.1 remains valid also for $\gamma \in \mathbb{R}$. Indeed, if $\gamma \leq 0$, then the equation

$$
\left[r^{-} \Phi\left(x^{\prime}\right)\right]^{\prime}+0 \cdot \Phi(x)=0
$$

is a non-oscillatory majorant of Eq. (4.3) (consider (4.1), (4.7)).

Since the asymptotic almost periodicity of a function $f$ implies the asymptotic almost periodicity of $|f|$ (consider, e.g., Corollary 2.1), we can also apply Theorem 4.1 in the case when the function $s$ in Eq. (4.3) changes its sign. More precisely, for Eq. (4.3) with $\gamma \in \mathbb{R}$ and asymptotically almost periodic functions $r$ and $s$, we obtain the following result by the Sturm comparison theorem. If $\inf \left\{r(t) ; t \in \mathbb{R}_{0}^{+}\right\}>0, \lim _{t \rightarrow \infty} s(t) \neq 0$, and $\gamma<q^{-p}\left(M\left(r^{1-q}\right)\right)^{1-p}(M(|s|))^{-1}$, then Eq. (4.3) is non-oscillatory. 
Let us illustrate the connection between (ordinary) half-linear differential equations analyzed in this paper and partial differential equations with $p$-Laplacian. We refer, e.g., to [38-41]. For example, consider the equation

$$
\operatorname{div}\left(A(x)\|\nabla u\|^{p-2} \nabla u\right)+\left\langle\vec{b}(x),\|\nabla u\|^{p-2} \nabla u\right\rangle+c(x) \Phi(u)=0,
$$

where $x \in \mathbb{R}^{n}, \vec{b}$ is a continuous non-zero $n$-vector function, $A$ is an elliptic $n \times n$ matrix function with differentiable components, $c$ is a Hölder continuous function, and div, $\nabla$, $\|\cdot\|$, and $\langle\cdot, \cdot\rangle$ stand for the divergence operator, the nabla operator, the Euclidean norm, and the usual scalar product in $\mathbb{R}^{n}$, respectively. Let $l>1, S(a)=\left\{x \in \mathbb{R}^{n},\|x\|=a\right\}$, $\mathrm{d} \sigma$ be the element of the surface of the sphere $S(t)$, and $\lambda_{\min }(x)$ be the smallest eigenvalue of the matrix $A(x)$. According to [40, Theorem 1], we have the following result. If the functions

$$
\begin{aligned}
& r(t)=\left(\frac{l}{l-1}\right)^{p-1} \int_{S(t)}\|A(x)\|^{p} \lambda_{\min }^{1-p}(x) \mathrm{d} \sigma, \\
& s(t)=\frac{t^{p}}{\gamma} \int_{S(t)}\left[c(x)-\frac{l^{p-1}}{\lambda_{\min }^{p-1}(x)} \frac{\|\vec{b}(x)\|^{p}}{p^{p}}\right] \mathrm{d} \sigma
\end{aligned}
$$

satisfy the requirements of Theorem 4.1 and $\gamma>\Gamma$ (see (4.2)), then Eq. (5.1) is oscillatory.

\section{Competing interests}

The authors declare that they have no competing interests.

\section{Authors' contributions}

The authors declare that the research was realized in collaboration with the same responsibility and contributions. Both authors read and approved the final manuscript.

\section{Author details}

'Department of Mathematics, Mendel University in Brno, Zemědělská 1, Brno, CZ-613 00, Czech Republic. ²Department of Mathematics and Statistics, Masaryk University, Kotlárská 2, Brno, CZ-611 37, Czech Republic.

\section{Acknowledgements}

The first author is supported by Grant P201/10/1032 of the Czech Science Foundation. The second author is supported by the Operational Programme 'Education for Competitiveness (POSTDOC II.)' under the project CZ.1.07/2.3.00/30.0037. The project is co-financed by the European Social Fund and the state budget of the Czech Republic.

\section{Received: 28 December 2012 Accepted: 17 April 2013 Published: 30 April 2013}

\section{References}

1. Beesack, PR: Hardy's inequality and its extensions. Pac. J. Math. 11(1), 39-61 (1961)

2. Elbert, Á: A half-linear second order differential equation. Colloq. Math. Soc. János Bolyai 30, 158-180 (1979)

3. Mirzov, JD: On some analogs of Sturm's and Kneser's theorems for nonlinear systems. J. Math. Anal. Appl. 53(2), 418-425 (1976)

4. Došlý, O, Řehák, P: Half-Linear Differential Equations. Elsevier, Amsterdam (2005)

5. Agarwal, RP, Grace, AR, O'Regan, D: Oscillation Theory for Second Order Linear, Half-Linear, Superlinear and Sublinear Dynamic Equations. Kluwer Academic, Dordrecht (2002)

6. Bihari, I: An oscillation theorem concerning the half-linear differential equation of second order. Magy. Tud. Akad. Mat. Kut. Intéz. Közl. 8, 275-280 (1964)

7. Elbert, A: The Wronskian and the half-linear differential equations. Studia Sci. Math. Hung. 15(1-3), 101-105 (1980)

8. Drábek, P: Fredholm alternative for the $p$-Laplacian: yes or no? In: Function Spaces, Differential Operators and Nonlinear Analysis (Pudasjärvi, 1999), pp. 57-64. Acad. Sci. Czech Rep., Prague (2000)

9. Díaz, J: Nonlinear Partial Differential Equations and Free Boundaries, Vol. 1: Elliptic Equations. Pitman, Boston (1985)

10. Cheban, DN: Asymptotically Almost Periodic Solutions of Differential Equations. Hindawi Publishing Corporation, New York (2009)

11. Corduneanu, C: Almost Periodic Oscillations and Waves. Springer, New York (2009)

12. Fink, AM: Almost Periodic Differential Equations. Springer, Berlin (1974)

13. Došlý, O, Hasil, P: Critical oscillation constant for half-linear differential equations with periodic coefficients. Ann. Mat. Pura Appl. 190(3), 395-408 (2011). doi:10.1007/s10231-010-0155-0

14. Hasil, P: Conditional oscillation of half-linear differential equations with periodic coefficients. Arch. Math. 44(2), 119-131 (2008) 
15. Kusano, T, Naito, Y: A oscillation and nonoscillation criteria for second order quasilinear differential equations. Acta Math. Hung. 76(1-2), 81-99 (1997). doi:10.1007/BF02907054

16. Schmidt, KM: Critical coupling constant and eigenvalue asymptotics of perturbed periodic Sturm-Liouville operators. Commun. Math. Phys. 211(2), 465-485 (2000). doi:10.1007/s002200050822

17. Schmidt, KM: Oscillation of perturbed Hill equation and lower spectrum of radially periodic Schrödinger operators in the plane. Proc. Am. Math. Soc. 127(8), 2367-2374 (1999). doi:10.1090/S0002-9939-99-05069-8

18. Gesztesy, F, Ünal, M: Perturbative oscillation criteria and Hardy-type inequalities. Math. Nachr. 189, 121-144 (1998). doi:10.1002/mana.19981890108

19. Hasil, P, Veselý, M: Critical oscillation constant for difference equations with almost periodic coefficients. Abstr. Appl. Anal. 2012, Article ID 471435 (2012). doi:10.1155/2012/471435

20. Krüger, H: On perturbations of quasiperiodic Schrödinger operators. J. Differ. Equ. 249(6), 1305-1321 (2010). doi:10.1016/j.jde.2010.06.018

21. Krüger, H, Teschl, G: Effective Prüfer angles and relative oscillation criteria. J. Differ. Equ. 245(12), 3823-3848 (2008), doi:10.1016/j.jde.2008.06.004

22. Krüger, H, Teschl, G: Relative oscillation theory for Sturm-Liouville operators extended. J. Funct. Anal. 254(6), 1702-1720 (2008). doi:10.1016/j.jfa.2007.10.007

23. Krüger, H, Teschl, G: Relative oscillation theory, weighted zeros of the Wronskian, and the spectral shift function. Commun. Math. Phys. 287(2), 613-640 (2009). doi:10.1007/s00220-008-0600-8

24. Došlý, O, Řezníčková, J: A remark on an oscillation constant in the half-linear oscillation theory. Appl. Math. Lett. 23(9), 971-974 (2010). doi:10.1016/j.aml.2010.04.019

25. Erbe, L: Oscillation results for second-order linear equations on a time scale. J. Differ. Equ. Appl. 8(11), 1061-1071 (2002). doi:10.1080/10236190290015317

26. Řehák, P: A critical oscillation constant as a variable of time scales for half-linear dynamic equations. Math. Slovaca 60(2), 237-256 (2010). doi:10.2478/s12175-010-0009-7

27. Řehák, P: On certain comparison theorems for half-linear dynamic equations on time scales. Abstr. Appl. Anal. 2004(7), 551-565 (2004). doi:10.1155/S1085337504306251

28. Řehák, P: New results on critical oscillation constants depending on a graininess. Dyn. Syst. Appl. 19(2), 271-287 (2010)

29. Dzurnak, A, Mingarelli, AB: Sturm-Liouville equations with Besicovitch almost-periodicity. Proc. Am. Math. Soc. 106(3) 647-653 (1989). doi:10.2307/2047418

30. Halvorsen, SG, Mingarelli, AB: On the oscillation of almost-periodic Sturm-Liouville operators with an arbitrary coupling constant. Proc. Am. Math. Soc. 97(2), 269-272 (1986). doi:10.2307/2046511

31. Staněk, S: A note on the oscillation of solutions of the differential equation $y^{\prime \prime}+\lambda q(t) y=0$ with an almost periodic coefficient. Acta Univ. Palacki. Olomuc., Fac. Rerum Nat., Math. 24(1), 67-70 (1985)

32. Li, HJ, Yeh, CC: An oscillation criterion of almost-periodic Sturm-Liouville equations. Rocky Mt. J. Math. 25(4), 1417-1429 (1995). doi:10.1216/rmjm/1181072154

33. Wong, FH, Yeh, CC: An oscillation criterion for Sturm-Liouville equations with Besicovitch almost-periodic coefficients. Hiroshima Math. J. 21(3), 521-528 (1991)

34. Sugie, J, Hata, S: Global asymptotic stability for half-linear differential systems with generalized almost periodic coefficients. Monatshefte Math. 166(2), 255-280 (2012). doi:10.1007/s00605-011-0297-1

35. Elbert, Á: Asymptotic behaviour of autonomous half-linear differential systems on the plane. Studia Sci. Math. Hung. 19(2-4), 447-464 (1984)

36. Kneser, A: Untersuchungen über die reellen Nullstellen der Integrale linearer Differentialgleichungen. Math. Ann. 42(3), 409-435 (1893). doi:10.1007/BF01444165

37. Veselý, M: Construction of almost periodic functions with given properties. Electron. J. Differ. Equ. 2011, 29 (2011)

38. Došlý, O, Mařík, R: Nonexistence of positive solutions of PDE's with p-Laplacian. Acta Math. Hung. 90(1-2), 89-107 (2001). doi:10.1023/A:1006739909182

39. Kusano, T, Jaroš, J, Yoshida, N: A Picone-type identity and Sturmian comparison and oscillation theorems for a class of half-linear partial differential equations of second order. Nonlinear Anal., Theory Methods Appl. 40(1-8), 381-395 (2000). doi:10.1016/S0362-546X(00)85023-3

40. Mařík, R: Ordinary differential equations in the oscillation theory of partial half-linear differential equation. J. Math. Anal. Appl. 338(1), 194-208 (2008). doi:10.1016/j.jmaa.2007.05.015

41. Naito, Y, Usami, H: Oscillation criteria for quasilinear elliptic equations. Nonlinear Anal., Theory Methods Appl. 46(5), 629-652 (2001). doi:10.1016/S0362-546X(00)00136-X 